\title{
Effectiveness of additional follow-up telephone counseling in a smoking cessation clinic in Beijing and predictors of quitting among Chinese male smokers
}

Lei Wu ${ }^{1,2}$, Yao He ${ }^{1,2,3^{*}}$, Bin Jiang ${ }^{4}$, Fang Zuo ${ }^{4}$, Qinghui Liu ${ }^{5}$, Li Zhang ${ }^{6}$, Changxi Zhou ${ }^{5}$, Miao Liu ${ }^{1,2}$, Hongyan Chen ${ }^{1,2}$, KK Cheng ${ }^{7}$, Sophia S. C. Chan ${ }^{8}$ and Tai Hing Lam ${ }^{9}$

\begin{abstract}
Background: No previous studies have investigated whether additional telephone follow-up counseling sessions after face-to-face counseling can increase quitting in China, and whether this strategy is feasible and effective for promoting smoking cessation is still unclear.

Methods: A non-randomized controlled study was conducted in Beijing. We compared the quit rates of one group which received face-to-face counseling (FC) alone (one session of $40 \mathrm{~min}$ ) to another group which received the same face-to-face counseling plus four follow-up sessions of brief telephone counseling (15-20 min each) at 1 week, 1, 3 and 6 month follow-up (FCF). No smoking cessation medication was provided. From October 2008 to August 2013, Chinese male smokers who sought treatment in a part-time regular smoking cessation clinic of a large general hospital in Beijing were invited to participate in the present study. Eligible male smokers $(n=547)$ were divided into two groups: FC $(n=149)$ and FCF $(n=398)$. Main outcomes were self-reported 7-day point prevalence and 6 month continuous quit rates at 12 month follow-up.
\end{abstract}

Results: By intention to treat, at 12 month follow-up, the 7-day point prevalence and 6 month continuous quit rates of FC and FCF were $14.8 \%$ and $26.4 \%$, and $10.7 \%$ and $19.6 \%$ respectively. The adjusted odds ratios (95\% confidence intervals) of quitting in FCF compared to FC was $2.34(1.34-4.10)(P=0.003)$ and $2.41(1.28-4.52)(P=0.006)$, respectively. Stepwise logistic regression showed that FCF, being married, unemployed and a lower Fagerström score were significant independent predictors of 6 month continuous quitting at 12 month follow-up.

Conclusions: Using systematically collected data from real-world practice, our smoking cessation clinic has shown that the additional telephone follow-up counseling sessions doubled the quit rate.

Keywords: Face-to-face counseling, Additional telephone follow-up counseling, Chinese male smoker, Quit rate, Predictors of quitting

\footnotetext{
* Correspondence: yhe301@x263.net

${ }^{1}$ Department of Epidemiology, Institute of Geriatrics, Chinese PLA General Hospital, 28 Fuxing Road, Beijing 100853, China

${ }^{2}$ Beijing Key Laboratory of Aging and Geriatrics, Chinese PLA General Hospital, Institute of Geriatrics, 28 Fuxing Road, Beijing 100853, China Full list of author information is available at the end of the article
} 


\section{Background}

China has one-third of the world's smokers, about 350 million in total. The 2010 Global Adult Survey reported that $52.9 \%$ of men and $2.4 \%$ of women were current smokers in China [1, 2]. To reduce the disease burden of tobacco worldwide, smoking cessation in China should play a critical role. However, in China Mainland, health care workers have little motivation to help smokers quit smoking [3]. Additionally, the effectiveness of existing smoking cessation interventions and smoking cessation services is still unclear $[3,4]$.

In western developed countries, studies which compared the effectiveness of various types of follow-up intervention (different frequency and intensity of counseling) after first counseling versus first counseling alone, have shown that the quit rates of smokers who received a "booster" (additional follow-up telephone counseling) were higher than those smokers who did not receive the "booster" [5-8]. After reviewing 22 Randomized Controlled Trials (RCTs), Pan et al. reported that the quit rate in the treatment group (with additional telephone counseling) was $64 \%$ greater than that in the comparison group [9]. According to a 2013 Cochrane systematic review, Stead et al. reported that telephone counseling as an adjunct to brief intervention or counseling increased quit rates, compared to brief intervention or counseling alone, and the relative risk (95\% confidence internal) was 1.4 (1.2-1.7) [10]. It is worth noting that most of the trials, which were included in both of the above reviews, were performed in western, developed highincome countries, but none of the included trials were conducted in China Mainland.

Moreover, one of the major challenges of smoking cessation is the high relapse rate. Among smokers who quit initially, up to $80 \%$ may relapse within a year [11]. By teaching coping skills and offering additional social support, the additional follow-up telephone counseling sessions might be an effective way to prevent relapse and to plan new quit attempts $[5,12]$. Sheffer et al. reported that the 6-month abstinence rate in the treatment group (telephone counseling including relapse prevention) was $20.9 \%$, which was significantly higher than that of the control group (without relapse prevention, $10.6 \%)$ [13].

Smoking cessation and cessation services are at an early stage of development in China Mainland. Both RCTs and observational studies about smoking cessation services are scarce. To our best knowledge, no previous studies have investigated whether additional telephone counseling sessions after face-to-face counseling can increase quitting in China, and whether this strategy is feasible and effective for promoting smoking cessation is still unclear. Although observational studies on smoking cessation interventions cannot provide the same degree of confidence for causal inference as RCTs, results from RCT often exaggerate the effect size because research subjects are carefully selected and are usually under exceptional care and attention [14]. Evaluation of real-world practice can provide more realistic effect size and can test the generalizability of RCT evidence when the interventions are implemented in different settings. When the results from a vigorous evaluation of an intervention in a new setting, such as in China, are consistent with RCT evidence elsewhere (which is predominantly from the West) and are widely disseminated, policy makers and health care professionals in similar settings are more likely to be motivated to implement the intervention.

Thus, we conducted a non-randomized controlled study in a real-world setting in one of the longest running smoking cessation clinics of Beijing, China. We aimed to compare the effect of one session face-to-face individual counseling plus follow-up telephone counseling with that of face-to-face counseling alone for Chinese male smokers in China Mainland. We also adjusted for the key potential confounders [15] so as to minimize the effect of confounding.

\section{Methods}

\section{Study setting}

This was a non-randomized controlled study which was based on the retrospective analysis of data collected systematically for the evaluation of the services in a smoking cessation clinic (SCC). We established a parttime SCC in the outpatient department of People's Liberation Army General Hospital (with 3400 hospital beds and on average about 10,000 out-patients per day) in Beijing. The SCC, modeled after the Hong Kong Smoking Cessation Health Centre [16], was aimed to serve as a new platform for cessation research and evaluation in China Mainland. In 1996, 22 smoking cessation clinics had been set up in Beijing. Ours is one of the very few clinics which are still running and treating smokers regularly [17]. It started operation from 22 October 2008, and the service is still operational at the time of submission of the present paper. We provided services in 4 weekday evenings (Monday to Thursday, from 6:30 to 9 p.m.) by eligible physicians. These physicians should meet the criteria as follows: (1) had got a medical degree, (2) had more than 5 years of relevant workplace experience, and (3) had completed a smoking cessation training program and passed the examinations [16]. The target clients were smokers who volunteered to seek treatment at our clinic and paid 7 yuan (about U.S. \$1) for the registration fee. No fees were charged for counseling. This study was approved by the Independent Ethics Committee of Chinese People's Liberation Army General Hospital (S2013-066-01). Signed informed consent was obtained from all eligible participants. 


\section{Subject recruitment and intervention}

The inclusion criteria were: (1) current smokers (smoked daily for at least 6 months at the time of survey) [18], Chinese, aged 18 years or above, and (2) agreed to participate in the follow-up and signed an informed consent form. The exclusion criteria were: (1) disagreed and did not sign the informed consent form, (2) did not conform to the definition of current smoker, (3) cognitively impaired (such as those participants who could not understand and complete the questionnaire reliably), and (4) serious deafness.

All smokers received the same intervention at the first visit. Before counseling, the smoker's smoking and related information was assessed using a baseline questionnaire through face-to-face interview lasting approximately $10 \mathrm{~min}$. Then the physician provided individual face-to-face counseling based on Prochaska's transtheoretical model [19] and the five 'A' (ask, advice, assess, assist and arrange) lasting at least $30 \mathrm{~min}$. The physician assessed the stage of readiness in quitting smoking, strengthened clients' motivation to quit smoking using the five ' $R$ ' (relevance, risks, rewards, roadblocks and repetition) approaches [20], and provided advice to overcome craving, psychological dependence and social-cultural factors associated with tobacco dependency [16]. No smoking cessation medication was provided.

After baseline intervention, smokers who visited our clinic from October 2008 to December $2010(n=254)$, had telephone follow-up by counselors at 1 week, 1, 3, 6 and 12 months. Our counselors were chosen from retired nurses, and they had completed a smoking cessation training program and passed the examinations. At follow-up, after assessing the smoking or quitting status, we added a "booster" (additional counseling). We asked whether the smokers or quitters had any problems. We provided problem-oriented suggestions or advice as appropriate, and also encouraged them to quit or maintain abstinence. Each follow-up lasted for about 15-20 min.

To study the effect of the "boosters", we could not do an RCT in the present real-world service situation in the clinic as random allocation of the smokers into two groups with different follow-up interventions could create confusion in the smokers as they came for a service and did not expect to be randomized. Because of the uncertainty of whether the "booster" was effective, we stopped the additional follow-up telephone counseling for all the smokers first counseled in 2011. These smokers had the same telephone follow-up assessment by trained counselors at 1, 3, 6 and 12 months with only questions about smoking and quitting, but with no further counseling. Each follow-up lasted for about 2-3 min. These smokers constituted the group of face-toface counseling only (FC, $n=149$ ).
After 2011, we resumed the additional follow-up telephone sessions for all smokers. The smokers from January 2012 to August $2013(n=144)$, together will those from October 2008 to December 2010 formed the face-to-face counseling plus follow-up telephone counseling group (FCF group, total $n=398$ ). The additional follow-up telephone sessions for all smokers were completed in August, 2014.

To enhance the integrity and quality of counseling, counselors were supervised during the entire project. Counselors called to contact the smokers of the two groups for at least 7 times at different days before considering them as lost to follow-up. Physicians who provided the baseline counseling were blinded to the subsequent grouping methods (with or without additional follow-up telephone sessions). The counselors who provided the additional follow-up telephone sessions could not be blinded to the grouping of the participants, but they did not know the aim of the present study, so that they could record the tobacco use status of smokers with minimal subjective bias.

\section{Data collection}

Data collection was done at the first visit and each followup interview using standardized and structured questionnaires [16, 21, 22]. The following baseline characteristics data of each participant were collected: gender, age, marital status, educational level, occupation and family income; tobacco related questions included smoking history, smoking status, place of smoking, past quitting history, motivation to quit, and perceived confidence, importance and difficulties in quitting smoking (all three based on the scale of $1-100$, denoting from the least to the most). All smokers had the Fagerström Test for Nicotine Dependence (FTND) and their dependence was classified as low (0-3), moderate (4-5) and severe (6-10) [23]. Exhaled carbon monoxide level was measured by trained technicians using a standard protocol and MicroCO [16]. Other questions included previous medical advice to quit, doctor diagnosed tobacco related chronic diseases and alcohol use. The follow-up questionnaires were similar to the baseline questionnaire, with deletion of redundant questions. We also added some questions on quitting as follows: "What was the date you started to stop smoking?", "How many times have you quit smoking for more than $24 \mathrm{~h}$ ?" and "When you quit smoking, did you have any withdrawal symptom?"

\section{Analysis}

The data were entered (double entry) using Epidata (3.1) and analyzed using SPSS (Inc., Chicago, IL, USA) for Windows (19.0). The baseline characteristics were described using descriptive statistics. The prevalence of quitters by different baseline factors was compared with chi-square 
test. We used the forward stepwise logistic regression to identify the independent predictors of successful quitting and to calculate adjusted odds rations (ORs) and $95 \%$ confidence intervals (CIs). Using intention to treat analysis (ITT), smokers who could not be contacted during follow-up were considered as non-quitters or nonreducers. Because the FCF group (28.1\%) had a much greater lost to follow-up rate than FC (18.8\%), and hence had greater percentages assumed to have had no improvement by ITT, complete case (per protocol) analysis was done by excluding those lost to follow-up as a sensitivity analysis. All $\mathrm{P}$ values were two-sided and $<0.05$ was considered as statistical significance.

The primary outcome of 7-day point prevalence quit rate was defined by not smoking any cigarettes during the past 7 days at 12 month follow-up, which was based on the United States Clinical Practice Guidelines [20]. The 6 month continuous quit rate was defined as not smoking any cigarettes during the past 6 months at 12 month follow-up. New quitters at 12 month followup was defined as still smoking cigarettes at 6 month follow-up, but not smoking any cigarettes for at least 7 days at 12 month follow-up. Relapse at 12 month follow-up was defined as not smoking any cigarettes at 6 month follow-up, but having smoked more than one cigarette each day for at least 7 days at 12 month followup. These were self-reported by the smokers without biochemical validation.

\section{Results}

From 22 October 2008 to 31 Aug 2013, the baseline sample included 570 eligible smokers. Because male smokers made up the majority of the smoking population in China [1], female smokers were few and had different characteristics from males, the present analysis included only 547 male smokers (149 in FC group and 398 in FCF group) and excluded 23 female smokers. Until 31 Aug 2014, 407 male smokers had completed the 12 month follow-up, 140 (25.6\%; $18.8 \%$ in FC and $28.1 \%$ in FCF) were lost to follow-up, mostly due to non-contacts (Fig. 1). These 140 smokers showed no differences from the 407 smokers in their baseline demographic characteristics, tobacco related and other factors (Additional file 1: Table S1).

\section{Demographic characteristics, tobacco related and other factors}

There were no statistically significant differences between the two groups, except that the FCF group perceived less difficulty in quitting and had more willingness to pay for quitting (Table 1). Most smokers were middle aged (mean age $=41.0$ years; $S D=11.3$ years), married, currently employed and well-educated. More than half smoked 20 cigarettes or more each day, started smoking before 18 and had been smoking for more than 20 years. More than three-quarters had experience of quitting and had no other smokers in the household. About $40 \%$ were severely dependent on nicotine with Fagerström score of 6-10 and were at the action stage of quitting. The mean exhaled carbon monoxide level was $12 \mathrm{ppm}$, and the mean score of perceived importance of, difficulty and confidence in quitting was 86,73 and 68 , respectively. More than half had doctor diagnosed tobacco related chronic diseases and perceived poor health status.

\section{Prevalence quit rates}

Table 2 and Additional file 1: Figure S1 show that by intention to treat, at 1, 3, 6 and 12 month follow-up, the 7 -day point prevalence quit rate of the FC group was stable, at $16.1 \%, 17.4 \%, 16.1 \%$ and $14.8 \%$, respectively. The rate in the FCF group rose steadily, from $18.6 \%$ at 1 month, $23.1 \%$ at 3 months, $25.9 \%$ at 6 months to $26.4 \%$ at 12 months. At 12 month follow-up, the 7-day point prevalence quit rate of the FCF group was significantly higher than that of the FC group, and the adjusted OR (95\% CI) was 2.34 (1.34-4.10), $P=0.003$. The 6 month continuous abstinence quit rate was $10.7 \%$ in FC and $19.6 \%$ in FCF, and the adjusted OR $(95 \% \mathrm{CI})$ was $2.41(1.28-4.52), P=0.006$.

The results on prevalence quit rates were quite similar by complete case analysis (Additional file 1: Table S2).

By intention to treat and complete case analysis, the quit rates at two different time periods (October 2008December 2010 and January 2012-August 2013) of FCF group at 1, 3, 6 and 12 month follow-up all showed no statistically significant differences (Additional file 1: Table S3), suggesting no period effect.

\section{Predictors of quitting}

Because cigarette consumption was a major item in Fagerström test, it was not included in the stepwise logistic regression model. Table 3 shows that grouping and Fagerström test score were both strong predictors of 7 day point and 6 month continuous quitting at 12 month follow-up.

For 6 month continuous abstinence quit rate, Fagerström test score was a strong predictor of quitting with a negative dose-response relationship. Compared to the score of $0-3$, the OR (95\% CI) of quitting for Fagerström score of 4-5 and 6-10 was $2.16(1.18-3.96)$ and 3.07 (1.78-5.32), respectively. FCF group $(2.17,1.21-3.90)$, being married $(2.79,1.15-6.79)$ and unemployed (1.80, 1.05-3.11) were also significant independent predictors of quitting.

Older age and having other smoker(s) in the household were also significant predictors of 7-day point quit rate at 12 month follow-up. 
By complete case analysis, the predictors of quitting were similar (Additional file 1: Table S4).

Additional file 1: Table S5 shows that at 3, 6 and 12 month follow-up, the new quit and relapse rates were: 3-month: FC $26.9 \%$ and $19.2 \%$, FCF $34.8 \%$ and $15.2 \%$; 6-month: FC $12.5 \%$ and $20.8 \%$, FCF $25.2 \%$ and $13.6 \%$; 12-month: FC $13.6 \%$ and $22.7 \%$, FCF $22.9 \%$ and $21.0 \%$, respectively. At 12 months, the relapse rates in the FC and FCF groups were similar but the new quit rate in the FCF group was almost two times that in the FCF group, which was not significant, possibly due to small numbers.

\section{Discussion}

Our SCC is one of the longest running part-time regular cessation clinics in China mainland where evidence on the effectiveness of various smoking cessation interventions is scarce. Using systematically collected data from real-world practice, we evaluated the effectiveness of combining one session face-to-face counseling (40 min) plus four telephone follow-up sessions of brief counseling (15-20 min each) as compared to the same face-to-face counseling alone (control group). Our study has provided new evidence of the effectiveness of the additional followup telephone sessions and identified predictors of

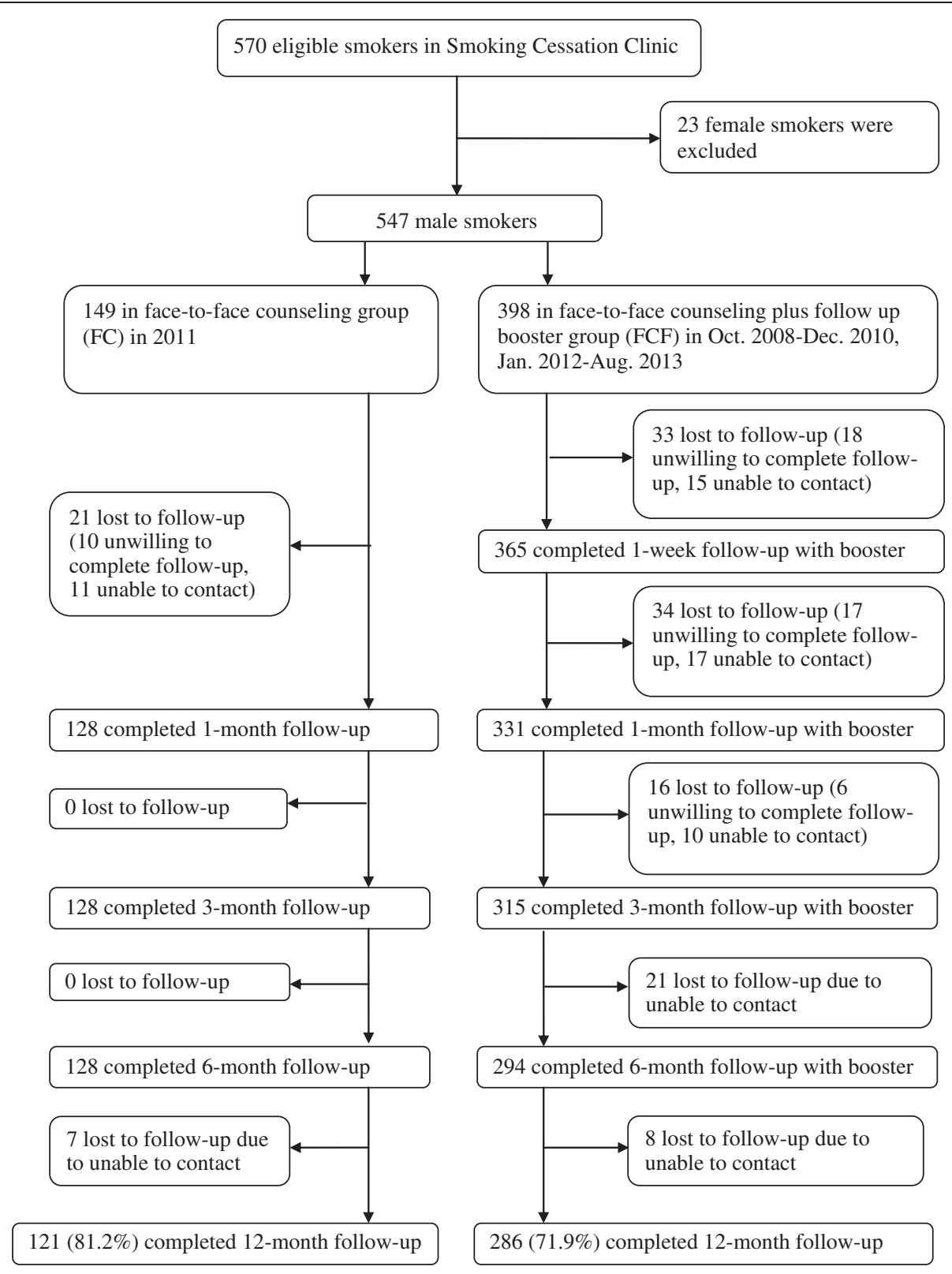

Fig. 1 Attrition flow chart 
Table 1 Demographic characteristics and tobacco-related factors of 547 male smokers in two groups

\begin{tabular}{|c|c|c|c|}
\hline & $F C(N=149)$ & FCF $(N=398)$ & $P$-value \\
\hline \multicolumn{4}{|l|}{ Demographic characteristics } \\
\hline Age (years) mean(SD) & $41.1(10.2)$ & $41.0(11.5)$ & \multirow[t]{2}{*}{0.89} \\
\hline Age (years) number (\%) & N (\%) & N (\%) & \\
\hline$<31$ & $22(14.8)$ & $78(19.6)$ & \multirow[t]{4}{*}{0.26} \\
\hline $31-40$ & $48(32.2)$ & $126(31.7)$ & \\
\hline $41-50$ & $54(36.2)$ & $114(28.6)$ & \\
\hline$>50$ & $25(16.8)$ & $80(20.1)$ & \\
\hline \multicolumn{4}{|l|}{ Marital status } \\
\hline Married & $134(89.9)$ & $345(86.7)$ & \multirow[t]{2}{*}{0.31} \\
\hline Single or divorced & $15(10.1)$ & $53(13.3)$ & \\
\hline \multicolumn{4}{|l|}{ Education } \\
\hline College and above & $93(62.4)$ & $232(58.3)$ & \multirow[t]{2}{*}{0.38} \\
\hline High school and below & $56(37.6)$ & $166(41.7)$ & \\
\hline \multicolumn{4}{|l|}{ Occupation } \\
\hline Currently employed & $118(79.2)$ & $318(79.9)$ & \multirow[t]{2}{*}{0.86} \\
\hline Student/unemployed/retired/others & $31(20.8)$ & $80(20.1)$ & \\
\hline \multicolumn{4}{|c|}{ Family income per month (Yuan, U.S.\$1 = 6 Yuan) } \\
\hline$<3000$ & $58(38.9)$ & $145(36.4)$ & \multirow[t]{3}{*}{0.70} \\
\hline $3000 \sim 6000$ & $37(24.8)$ & $113(28.4)$ & \\
\hline$>6000$ & $54(36.2)$ & $140(35.2)$ & \\
\hline \multicolumn{4}{|l|}{ Tobacco related factors } \\
\hline \multicolumn{4}{|l|}{ Age at initiation of smoking (years) } \\
\hline$<18$ & $48(32.2)$ & $135(33.9)$ & \multirow[t]{2}{*}{0.71} \\
\hline$\geq 18$ & $101(67.8)$ & $263(66.1)$ & \\
\hline \multicolumn{4}{|l|}{ Cigarettes smoked on average daily (cig/d) } \\
\hline$\geq 20$ & $96(64.4)$ & $241(60.6)$ & \multirow[t]{3}{*}{0.70} \\
\hline $10-19$ & $40(26.8)$ & $117(29.4)$ & \\
\hline$<10$ & $13(8.7)$ & $40(10.1)$ & \\
\hline \multicolumn{4}{|l|}{ Smoking duration (years) } \\
\hline$<20$ & 59 (39.6) & $175(44.0)$ & \multirow[t]{2}{*}{0.36} \\
\hline$\geq 20$ & $90(60.4)$ & $223(56.0)$ & \\
\hline \multicolumn{4}{|l|}{ Prior attempts to quit smoking } \\
\hline 0 & $33(22.1)$ & $102(25.6)$ & \multirow[t]{2}{*}{0.40} \\
\hline$\geq 1$ & $116(77.9)$ & $296(74.4)$ & \\
\hline \multicolumn{4}{|l|}{ Fagerström test score } \\
\hline Severe $(6-10)$ & $65(43.6)$ & $181(45.5)$ & \multirow[t]{3}{*}{0.41} \\
\hline Moderate (4-5) & $42(28.2)$ & $91(22.9)$ & \\
\hline Low (0-3) & $42(28.2)$ & $126(31.7)$ & \\
\hline \multicolumn{4}{|c|}{ Exhaled CO level at first visit (mean:12 ppm) } \\
\hline$\geq 12$ & $69(46.3)$ & $193(48.5)$ & \multirow[t]{2}{*}{0.65} \\
\hline$<12$ & $80(53.7)$ & $205(51.5)$ & \\
\hline \multicolumn{4}{|l|}{ Stage of quitting smoking } \\
\hline Contemplation & $43(28.9)$ & $91(22.9)$ & 0.35 \\
\hline Preparation & $51(34.2)$ & $146(36.7)$ & \\
\hline
\end{tabular}


Table 1 Demographic characteristics and tobacco-related factors of 547 male smokers in two groups (Continued)

\begin{tabular}{|c|c|c|c|}
\hline Action & $55(36.9)$ & $161(40.5)$ & \\
\hline \multicolumn{4}{|l|}{ Perceived importance of quitting (mean score:86) } \\
\hline$<86$ & $63(42.3)$ & $165(41.5)$ & \multirow[t]{2}{*}{0.86} \\
\hline$\geq 86$ & $86(57.7)$ & $233(58.5)$ & \\
\hline \multicolumn{4}{|l|}{ Perceived difficulty in quitting (mean score:73) } \\
\hline$\geq 73$ & $108(72.5)$ & $209(52.5)$ & \multirow[t]{2}{*}{$<0.001$} \\
\hline$<73$ & $41(27.5)$ & $189(47.5)$ & \\
\hline \multicolumn{4}{|l|}{ Perceived confidence in quitting (mean score:68) } \\
\hline$<68$ & $65(43.6)$ & $184(46.2)$ & \multirow[t]{2}{*}{0.59} \\
\hline$\geq 68$ & $84(56.4)$ & $214(53.8)$ & \\
\hline \multicolumn{4}{|l|}{ Expenditure on cigarettes per day, Yuan (mean:20) } \\
\hline$<20$ & $76(51.0)$ & $185(46.5)$ & \multirow[t]{2}{*}{0.35} \\
\hline$\geq 20$ & $73(49.0)$ & $213(53.5)$ & \\
\hline \multicolumn{4}{|l|}{ Willingness to pay for quitting, Yuan (mean:2000) } \\
\hline$<2000$ & $89(59.7)$ & $198(49.7)$ & \multirow[t]{2}{*}{0.04} \\
\hline$\geq 2000$ & $60(40.3)$ & $200(50.3)$ & \\
\hline \multicolumn{4}{|l|}{ Perceived health status at the first visit } \\
\hline Fair / poor / very poor & $93(62.4)$ & $270(67.8)$ & \multirow[t]{2}{*}{0.23} \\
\hline Very good / good & $56(37.6)$ & $128(32.2)$ & \\
\hline \multicolumn{4}{|l|}{ Number of other smokers in household } \\
\hline 0 & $123(82.6)$ & $323(81.2)$ & \multirow[t]{2}{*}{0.71} \\
\hline$\geq 1$ & $26(17.4)$ & $75(18.8)$ & \\
\hline Medical advice to quit & $48(32.2)$ & $139(34.9)$ & 0.55 \\
\hline Had doctor diagnosed tobacco related chronic diseases & $75(50.3)$ & $215(54.0)$ & 0.44 \\
\hline Current drinkers & $106(71.1)$ & $274(68.8)$ & 0.60 \\
\hline
\end{tabular}

Table 2 By intention to treat, quit rates of two groups at 1, 3, 6 and 12 month follow-up in 547 male smokers

\begin{tabular}{|c|c|c|c|c|c|}
\hline & FC N (\%) & FCF N (\%) & $\begin{array}{l}\text { Crude OR } \\
(95 \% \text { Cl) P-value }\end{array}$ & $\begin{array}{l}\text { Adjusted OR }{ }^{a} \\
(95 \% \text { Cl) } P \text {-value }\end{array}$ & $\begin{array}{l}\text { Adjusted OR }{ }^{b} \\
(95 \% \mathrm{Cl}) P \text {-value }\end{array}$ \\
\hline & $(N=149)$ & $(N=398)$ & & & \\
\hline \multicolumn{6}{|l|}{1 month follow-up } \\
\hline 7-day point prevalence & $24(16.1)$ & 74 (18.6) & $1.19(0.72-1.97) 0.50$ & $1.02(0.60-1.73) 0.94$ & $1.05(0.60-1.85) 0.86$ \\
\hline \multicolumn{6}{|l|}{3 month follow-up } \\
\hline 7-day point prevalence & $26(17.4)$ & $92(23.1)$ & $1.42(0.88-2.31) 0.15$ & $1.30(0.79-2.14) 0.31$ & $1.35(0.79-2.31) 0.27$ \\
\hline 1 month continuous abstinence & $23(15.4)$ & $73(18.3)$ & $1.23(0.74-2.05) 0.43$ & $1.11(0.65-1.88) 0.71$ & $1.17(0.67-2.05) 0.59$ \\
\hline \multicolumn{6}{|l|}{6 month follow-up } \\
\hline 7-day point prevalence & $24(16.1)$ & $103(25.9)$ & $1.82(1.11-2.97) 0.02$ & $1.70(1.03-2.82) 0.04$ & $1.92(1.12-3.32) 0.02$ \\
\hline 1 month continuous abstinence & $23(15.4)$ & $95(23.9)$ & $1.72(1.04-2.83) 0.03$ & $1.64(0.98-2.74) 0.06$ & $1.82(1.05-3.16) 0.03$ \\
\hline 3 month continuous abstinence & $22(14.8)$ & $77(19.3)$ & $1.39(0.83-2.32) 0.22$ & $1.31(0.77-2.24) 0.32$ & $1.48(0.83-2.62) 0.19$ \\
\hline \multicolumn{6}{|l|}{12 month follow-up } \\
\hline 7-day point prevalence & $22(14.8)$ & $105(26.4)$ & $2.07(1.25-3.43) 0.005$ & $2.08(1.24-3.50) 0.006$ & $2.34(1.34-4.10) 0.003$ \\
\hline 6 month continuous abstinence & $16(10.7)$ & 78 (19.6) & $2.03(1.14-3.60) 0.02$ & $2.14(1.19-3.88) 0.01$ & $2.41(1.28-4.52) 0.006$ \\
\hline
\end{tabular}

Face-to-face counseling plus follow-up telephone counseling, FCF; Face-to-face counseling only, FC; Odds ratio, OR; Confidence Interval, Cl

${ }^{a}$ Adjusted for demographic characteristics, perceived difficulty of quitting, willingness to pay for quitting and year of the first visit

${ }^{\mathrm{b}}$ Adjusted for all factors in Table 1 (with the exception of cigarette consumption) and year of the first visit 
Table 3 By intention to treat, logistic regression (stepwise) analysis for adjusted OR for predictors of quitting at 12 month follow-up

\begin{tabular}{|c|c|c|c|}
\hline Predictors & Adjusted OR $(95 \% \mathrm{Cl})^{\mathrm{a}}$ & $P$-value & $P$ for trend \\
\hline \multicolumn{4}{|l|}{ 7-day point prevalence } \\
\hline \multicolumn{4}{|l|}{ Group } \\
\hline$F C(N=149)$ & 1.00 & & \\
\hline FCF $(N=398)$ & $2.12(1.26-3.56)$ & 0.005 & \\
\hline \multicolumn{4}{|l|}{ Fagerström test score } \\
\hline Severe $(6-10)$ & 1.00 & & $<0.001$ \\
\hline Moderate (4-5) & $1.79(1.03-3.10)$ & 0.04 & \\
\hline Low (0-3) & $3.02(1.86-4.90)$ & $<0.001$ & \\
\hline \multicolumn{4}{|l|}{ Age (years) } \\
\hline$<31$ & 1.00 & & 0.02 \\
\hline $31-40$ & $2.20(1.16-4.19)$ & 0.02 & \\
\hline $41-50$ & $1.32(0.67-2.62)$ & 0.43 & \\
\hline$>50$ & $2.49(1.24-5.00)$ & 0.01 & \\
\hline \multicolumn{4}{|l|}{ Number of other smokers in household } \\
\hline 0 & 1.00 & & \\
\hline$\geq 1$ & $1.81(1.07-3.05)$ & 0.03 & \\
\hline \multicolumn{4}{|l|}{6 month continuous abstinence } \\
\hline \multicolumn{4}{|l|}{ Group } \\
\hline$F C(N=149)$ & 1.00 & & \\
\hline FCF $(N=398)$ & $2.17(1.21-3.90)$ & 0.01 & \\
\hline \multicolumn{4}{|l|}{ Fagerström test score } \\
\hline Severe $(6-10)$ & 1.00 & & $<0.001$ \\
\hline Moderate (4-5) & $2.16(1.18-3.96)$ & 0.01 & \\
\hline Low (0-3) & $3.07(1.78-5.32)$ & $<0.001$ & \\
\hline \multicolumn{4}{|l|}{ Marital status } \\
\hline Single or divorced & 1.00 & & \\
\hline Married & $2.79(1.15-6.79)$ & 0.02 & \\
\hline \multicolumn{4}{|l|}{ Occupation } \\
\hline Currently employed & 1.00 & & \\
\hline Student/unemployed/retired/others & $1.80(1.05-3.11)$ & 0.03 & \\
\hline
\end{tabular}

Face-to-face counseling plus follow-up telephone counseling, FCF Face-to-face counseling only, FC Odds ratio, OR Confidence Interval CI

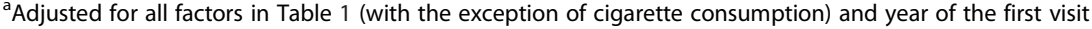

successful quitting in Chinese male smokers. Our experience and findings have demonstrated the feasibility and acceptability of such interventions, and the benefits of developing a 'model' smoking cessation clinic as a platform for research and development.

By intention to treat, at 12 month follow-up, the 7-day point prevalence and 6 month continuous quit rate of FC and FCF were $14.8 \%$ and $26.4 \%$, and $10.7 \%$ and $19.6 \%$, respectively. Among the 320 million ever smokers in China, only less than $4 \%$ had stopped smoking for at least 2 years [18]. The much higher quit rates in our clinic could be due to the stronger motivation to quit in smokers actively seeking help from a clinic. Our quit rates could serve as references for non-pharmacological interventions in other smoking cessation clinics in China.

Our quit rates, with no medications, appeared to be quite similar to many other RCTs. An RCT in Germany showed that the 7-day point prevalence quit rate of the group without additional follow-up telephone sessions was $16.8 \%$, and that of the group with additional follow-up telephone sessions was $30.3 \%$ at 6 month follow-up [5]. Zhu et al. reported that the 1 month continuous abstinence quit rate at 6 month follow-up was $14.1 \%$ in 5 sessions with two additional follow-up sessions compared to $7.6 \%$ with self-help booklets alone [7]. In the California Cancer Center, Berndt et al. 
reported that the 12-month continuous abstinence rates for those who made a quit attempt were $14.7 \%$ for the self-help quit kit group and $26.7 \%$ for the group with the self-help quit kit plus 6 telephone counseling sessions [6].

At 1, 3, 6 and 12 month follow-up, the 7-day point prevalence quit rate of our FC group was relatively stable, but the rate in the FCF group increased steadily. Our 12 month 7-day point prevalence quit rate and 6 month continuous abstinence rate were higher in the FCF than FC group (the adjusted OR and $95 \% \mathrm{CI}$ was 2.08, 1.24-3.50 and 2.14, $1.19-3.88$, respectively). By complete case analysis, as expected, the differences were greater and more significant, suggesting greater effectiveness of FCF. These, together with the results from comparing new quit rates and relapse rates, indicated that the additional follow-up telephone sessions had some effect on late-onset quitting, mainly by promoting more new quitting and preventing a few relapses. Prospective studies with a larger sample size are needed to examine late-onset quitting and smoking relapse prevention.

We identified that smokers who had lower nicotine dependence, were older, married or unemployed were more likely to quit in the present study. Smokers with lower nicotine dependence had little withdrawal symptoms, and thus they can quit more easily than those smokers with higher nicotine dependences [24-29]. Smokers with advancing age have increased concern about their health status and adverse health consequences of tobacco use, and thus they often have more intention to quit smoking [24, 27-30]. Given that older smokers are often more motivated to quit, the older age of the unemployed group (including the retired) may have contributed to this finding. Married smokers were more likely to have stronger social support to remain abstinent than the unmarried ones [27].

The present study had several limitations. Firstly, an important limitation was selection bias since the smokers were not assigned randomly to the FC and FCF group. We did not do a sample size calculation as the quit rates were unpredictable. But there were no important statistically significant differences of demographic characteristics and tobacco related factors between these two groups, as they sought treatment voluntarily from our SCC. Secondly, because our study lasted for about 6 years, possible time period effect might have affected our comparison, although we had adjusted for the variable of 'year of the first visit'. Thirdly, although the counselors did not know the aim of the research, social desirability bias might be present. The intervention group received more attention from the counseling, and thus might have been more inclined to falsely report abstinence. Fourthly, there was a large difference in the loss to follow-up rates between the two groups (18.8 \% in FC vs. $28.1 \%$ in FCF). One possible explanation was that smokers who received the additional follow-up counseling sessions (FCF group) were required to spend more time than the FC group. Smokers with less motivation to quit smoking were more likely to refuse to receive the additional follow-up counseling because they might consider the additional counseling useless. Additionally, both intention to treat analysis and complete case (per protocol) analysis showed that the quit rates of FCF group were significantly higher than the FC group, which indicated that the large difference in the loss to follow-up rates did not substantially affect the results. Finally, about $65 \%$ of our smokers came from outside of Beijing, and thus it was not convenient for them to return to our clinic for a face-to-face follow-up interview. Of the few (about $9 \%$ ) who came back eventually, their exhaled carbon monoxide and saliva cotinine (measured by NicAlert) confirmed the quitting status of over $95 \%$.

\section{Conclusion}

Our study has shown that the service of one session faceto-face counseling plus four telephone follow-up sessions of brief "booster" counseling for smoking cessation is feasible and acceptable to Chinese smokers. To the best of our knowledge, we have provided the first evidence from a smoking cessation clinic in China or any developing countries that regular additional follow-up telephone problemoriented counseling session can increase the effectiveness of a face-to-face counseling session alone. With systematic data collection, regular follow-up and rigorous evaluation, the new evidence and knowledge generated by our SCC can serve as a model to guide future smoking cessation service developments in China mainland and other middleand low-income countries.

\section{Additional file}

Additional file 1: Table S1. Demographic characteristics and tobacco related factors of 407 completed and 140 lost to 12 month follow-up. Table S2. By complete case (per protocol) analysis, quit rates of two groups at 1, 3, 6 and 12 month follow-up in 407 male smokers. Table S3. By intention to treat and complete case (per protocol) analysis, quit rates of two different time period of FCF group smokers at 1, 3, 6 and 12 month follow-up. Table S4. By complete case (per protocol) analysis, logistic regression (stepwise) analysis for adjusted OR for predictors of quitting at 12 month follow-up. Table S5. 7-day point prevalence relapse and new quit rate at 3, 6 and 12 month follow-up in two groups. Figure S1. Quit rates of two groups at 1, 3, 6 and 12 month follow-up, by intention to treat and complete case (per protocol) analysis. (DOC $320 \mathrm{~kb}$ )

\section{Competing interests}

All authors declare that they have no competing interests.

Authors' contributions

$\mathrm{YH}, \mathrm{BJ}$ and $\mathrm{THL}$ helped design the study and analyzed the data. BJ, FZ, QHL, CXZ and LZ helped data collection and field operations. THL and SSCC contributed to the setting up of the clinic, design of the interventions and provided training. All authors helped prepare the manuscript. We thank $L X, J H Z$ and JF for research assistance in the interview of follow-up. All authors read and approved the final manuscript. 


\section{Acknowledgments}

This study was supported by research grants from the National Natural Science Foundation of China, 81373080; Beijing Municipal Science and Technology Commission, Z121107001012070; Cancer Research UK: Smoking cessation and advocacy training among health professionals in Beijing and Guangzhou, China, C1343/A10155.

\section{Author details}

'Department of Epidemiology, Institute of Geriatrics, Chinese PLA General Hospital, 28 Fuxing Road, Beijing 100853, China. ${ }^{2}$ Beijing Key Laboratory of Aging and Geriatrics, Chinese PLA General Hospital, Institute of Geriatrics, 28 Fuxing Road, Beijing 100853, China. ${ }^{3}$ State Key Laboratory of Kidney Disease, Chinese PLA General Hospital, 28 Fuxing Road, Beijing 100853, China. ${ }^{4}$ Nanlou Faculty of Clinical Medicine, Department of Acupuncture, Chinese PLA General Hospital, 28 Fuxing Road, Beijing 100853, China. ${ }^{5}$ Nanlou Faculty of Clinical Medicine, Department of Respiration, Chinese PLA General Hospital, 28 Fuxing Road, Beijing 100853, China. ${ }^{6}$ Nanlou Faculty of Clinical Medicine, Department of Rehabilitation, Chinese People's Liberation Army General Hospital, Beijing, China. ${ }^{7}$ Public Health, Epidemiology and Biostatistics, University of Birmingham, Birmingham, UK. ${ }^{8}$ School of Nursing, The University of Hong Kong, Hong Kong, China. ${ }^{9}$ Department of Community Medicine and School of Public Health, The University of Hong Kong, Hong Kong, China.

Received: 7 February 2015 Accepted: 8 January 2016

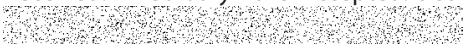

\section{References}

1. World Health Organization. China wrestles with tobacco control. An interview with Dr Yang Gonghuan. Retrieved from http://www.who.int/ bulletin/volumes/88/4/10-040410/en/index.html. 2010.

2. Yang GH, Li Q, Wang CX, Hsia J, Yang Y, Xiao L, et al. Findings from 2010 Global Adult Tobacco Survey: implementation of MPOWER policy in China. Biomed Environ Sci. 2010;23(6):422-9.

3. Jiang Y, Ong MK, Tong EK, Yang Y, Nan Y, Gan Q, et al. Chinese physicians and their smoking knowledge, attitudes, and practices. Am J Prev Med. 2007:33(1):15-22.

4. Gruder CL, Trinidad DR, Palmer PH, Xie B, Li L, Johnson CA. Tobacco smoking, quitting, and relapsing among adult males in Mainland China: the China Seven Cities Study. Nicotine Tob Res. 2013;15(1):223-30

5. Metz K, Flöter S, Kröger C, Donath C, Piontek D, Gradl S. Telephone booster sessions for optimizing smoking cessation for patients in rehabilitation centers. Nicotine Tob Res. 2007;9(8):853-63.

6. Berndt N, Bolman C, Froelicher ES, Mudde A, Candel M, de Vries H, et al. Effectiveness of a telephone delivered and a face-to-face delivered counseling intervention for smoking cessation in patients with coronary heart disease: a 6-month follow-up. J Behav Med. 2014;37(4):709-24

7. Zhu SH, Stretch V, Balabanis M, Rosbrook B, Sadler G, Pierce JP. Telephone counseling for smoking cessation: effects of single-session and multiplesession interventions. J Consult Clin Psychol. 1996;64(1):202-11.

8. Rabius V, Pike K, Hunter J, Wiatrek D, McAlister AL. Effects of frequency and duration in telephone counselling for smoking cessation. Tob Control. 2007; 16 Suppl 1:i71-4

9. Pan W. Proactive telephone counseling as an adjunct to minimal intervention for smoking cessation: a meta-analysis. Health Educ Res. 2006; 21(3):416-27.

10. Stead LF, Hartmann-Boyce J, Perera R, Lancaster T. Telephone counselling for smoking cessation. Cochrane Database Syst Rev. 2013;8:CD002850.

11. Centers for Disease Control and Prevention. Cigarette smoking-attributable mortality and years of potential life lost-United States, 1990. JAMA. 1993; 270(12):1408,1410,1413.

12. Brandon $T H$, Collins BN, Juliano LM, Lazev AB. Preventing relapse among former smokers: A comparison of minimal interventions through telephone and mail. J Consult Clin Psychol. 2000;68(1):103-13.

13. Sheffer CE, Stitzer M, Brandon T, Bursac Z. Effectiveness of adding relapse prevention materials to telephone counseling. J Subst Abuse Treat. 2010; 39(1):71-7.

14. Kotz D, Brown J, West R. 'Real-world' effectiveness of smoking cessation treatments: a population study. Addiction. 2014:109(3):491-9.
15. Brose LS, McEwen A, West R. Does it matter who you see to help you stop smoking? Short-term quit rates across specialist Stop Smoking Practitioners in England. Addiction. 2012;107(11):2029-36.

16. Abdullah AS, Hedley AJ, Chan SS, Ho WW, Lam TH. Hong Kong council on smoking and health Smoking Cessation Health Centre $(\mathrm{SCHC})$ steering group. Establishment and evaluation of a smoking cessation clinic in Hong Kong: a model for the future service provider. J Public Health (Oxf). 2004; 26(3):239-44.

17. Wu X, Yang Y, Jiang $Y$. The development of smoking cessation clinic in China. Health Research. 2008;38:50-2 [article in Chinese].

18. Yang G, Ma J, Chen A, Zhang Y, Samet JM, Taylor CE, et al. Smoking cessation in China: findings from the 1996 national prevalence survey. Tob Control. 2001;10(2):170-4.

19. Prochaska JO, Goldstein MG. Process of smoking cessation. Implications for clinicians. Clin Chest Med. 1991:12(4):727-35.

20. The Tobacco Use and Dependence Clinical Practice Guideline Panel, Staff, and Consortium Representatives. A clinical practice guideline for treating tobacco use and dependence: a US Public Health Service report. The tobacco use and dependence clinical practice guideline panel, staff, and consortium representatives. JAMA. 2000;283(24):3244-54.

21. Chan SS, Leung DY, Wong DC, Lau CP, Wong VT, Lam TH. A randomized controlled trial of stage-matched intervention for smoking cessation in cardiac out-patients. Addiction. 2012;107(4):829-37.

22. Lam TH, Abdullah AS, Chan SS, Hedley AJ. Hong Kong council on smoking and health Smoking Cessation Health Centre (SCHC) steering group. Adherence to nicotine replacement therapy versus quitting smoking among Chinese smokers: a preliminary investigation. Psychopharmacology(Berl). 2005;177(4):400-8.

23. Fagerström KO. Measuring degree of physical dependence to tobacco smoking with reference to individualization of treatment. Addict Behav. 1978:3(3-4):235-41.

24. Stolz D, Scherr A, Seiffert B, Kuster M, Meyer A, Fagerström KO, et al. Predictors of Success for Smoking Cessation at the Workplace: A Longitudinal Study. Respiration. 2014;87(1):18-25.

25. Bhang SY, Choi SW, Ahn JH, Kim K, Kim H, Park HK. Predictors of success at six-month follow-up at a public smoking cessation clinic in South Korea. Asia Pac Psychiatry. 2013:5(3):197-204

26. Carreras Castellet JM, Maldonado Aróstegui B, Quesada Laborda M, Sánchez Sánchez B, De La Puerta IN, Sánchez Agudo L. Telephone-based smoking cessation. Predictors of success. Med Clin (Barc). 2012;138(6):242-5.

27. Wenig JR, Erfurt L, Kröger CB, Nowak D. Smoking cessation in groups-who benefits in the long term? Health Educ Re. 2013;28(5):869-78.

28. Steinberg MB, Foulds J, Richardson DL, Burke MV, Shah P. Pharmacotherapy and smoking cessation at a tobacco dependence clinic. Prevent Med. 2006; 42(2):114-9.

29. Osler M, Prescott E. Psychosocial, behavioral, and health determinants of successful smoking cessation: a longitudinal study of Danish adults. Tob Control. 1998:7(3):262-7.

30. Monsó E, Campbell J, Tønnesen P, Gustavsson G, Morera J. Sociodemographic predictors of success in smoking intervention. Tob Control. 2001;10(2):165-9.

\section{Submit your next manuscript to BioMed Central and we will help you at every step:}

- We accept pre-submission inquiries

- Our selector tool helps you to find the most relevant journal

- We provide round the clock customer support

- Convenient online submission

- Thorough peer review

- Inclusion in PubMed and all major indexing services

- Maximum visibility for your research

Submit your manuscript at www.biomedcentral.com/submit 